\title{
Non-thermal plasma treatment altered gene expression profiling in non-small-cell lung cancer A549 cells
}

Jue Hou ${ }^{1 \dagger}$, Jie Ma ${ }^{1,2 \dagger}$, K. N. Yu ${ }^{1,4}$, Wei Li ${ }^{1}$, Cheng Cheng ${ }^{3}$, Lingzhi Bao ${ }^{1,5^{*}}$ and Wei Han ${ }^{1,5^{*}}$

\begin{abstract}
Background: Recent technological advances in atmospheric plasmas have made the creation of non-thermal atmospheric pressure plasma (NTP) possible for utilization in the medical field. Although accumulated evidence suggests that NTP induces cell death in various cancer cell types thus offering a promising alternative treatment strategy, the mechanism underlying its therapeutic effect is not fully understood.

Results: We analyzed relevant signaling cascades associated with the tumor protein p53, in particular the cell cycle arrest, DNA damage as well as the underlying apoptosis pathways. Based on our results, the major effect from plasma exposure was found to be the activation of MAPK and p53 signaling pathways, resulting in changes in gene expression of MEKK, GADD, FOS and JUN. Finally, a significant modulation in expression of genes related to cellular proliferation and differentiation was observed.

Conclusion: Overall, the presented data of the tumor transcriptome helped identify the key players in modulated gene expression following exposure to plasma at the molecular level, and also helped interpret the downstream processes. The present work laid the foundation for further studies to clarify the roles of multiple pathways in plasma-induced biological processes. Further investigation of these genes in other cell lines may reveal comprehensive mechanisms of plasma induced effects.
\end{abstract}

Keywords: Non-thermal plasma, Gene profiling, NSCLC A549 cell line, Multiple signal pathways

\section{Background}

Plasma known as the fourth state of matter is partially or completely ionized gas including a mixture of electrons, ions, radicals, and energetic photons, which is typically generated under high temperature conditions. Due to the interdisciplinary effort of physics and biotechnology, recent technological progress in atmospheric plasmas has led to the creation of cold plasmas with an ion temperature close to the room temperature, which can be controlled or changed according to the desired applications. Moreover, that plasma can interact with organic materials without causing thermal damage to the cell

\footnotetext{
* Correspondence: lingzhibao@hfcas.ac.cn; hanw@hfcas.ac.cn ${ }^{1}$ Center of Medical Physics and Technology, Hefei Institutes of Physical Science, Chinese Academy of Sciences, Hefei, China

Full list of author information is available at the end of the article
}

surface, and can effectively inactivate different kinds of pathogens without adversely affecting the healthy tissues. Many other biological applications have also been explored. Accumulated evidence has shown that plasma can be generated and play an increasingly important role in various applications at room temperature, such as blood coagulation, wound healing, and tissue and device sterilization [1]. Furthermore, using endoscopic effect of non-thermal atmospheric pressure plasma has been developed in both clinical applications and disinfection of medical devices [2, $3]$, as a targeted and low invasive technique. Additionally, some researches have been performed in the utility of cold plasma for anti-tumor or cancer therapy in various tumor 
cell lines including several solid malignant (cervical carcinoma, lung carcinoma, glioblastoma, thyroid carcinoma, oral carcinoma and colorectal carcinoma) cells [4-6] and lymphoma cells $[7,8]$. In particular, it is remarked here that nonthermal atmospheric pressure plasma (NTP) could treat some tumors under the superficial skin such as melanoma, as well as head and neck cancers $[9,10]$.

Moreover, accumulated evidence has revealed that plasma treatment can induce various effects on multiple cancer cell types, including oxidative stress such as ROS and RNS mediated mitochondria-dependent apoptosis $[1,6,11]$, cell cycle arrest, cell growth inhibition [1] and obstructed tumor invasion [12]. The relationships between plasma exposure and cellular responses have been partially established in recent studies which demonstrated that plasma treatment of cells resulted in intense alterations of apoptotic signaling $[9,13]$ related transcription factors [14], DNA damages [8, 13] and ATM/ p53 [9] pathways. In particular, the reactive oxygen/nitrogen species including $\mathrm{H}_{2} \mathrm{O}_{2}, \mathrm{Ox}, \mathrm{NO}_{2}, \mathrm{NO}_{\mathrm{x}}$, which lead to depolarization of the mitochondrial membrane and mitochondrial ROS accumulation that displayed a compromised redox status evident from increased $\mathrm{NADP}+/ \mathrm{NADPH}$ levels, reduced the GSH/GSSG ratios and enhanced cytogenetic damages revealed by micronucleus formation assessment [7, 8]. Moreover, Yan and colleagues elaborated on the several stages involved in the induction of cancer cell death by cold atmosphericpressure plasma through increased concentrations of NO, ROS and lipid peroxide [15]. In fact, more factors affected the plasma effect on cancer cell viability. Chen defined the "plasma dosage" which characterized the relationship between the characteristics of the cold plasma (including treatment duration, input/output voltage, flow rate and composition of feed gas) and cell viability [4]. In reality, the capability of cell killing by plasma was even found to be sensitive to the concentration of fetal bovine serum in the media as well as the storage temperature for the media [16].

However, the detailed mechanisms underlying these effects have not yet been fully identified. An original research indicated that NTP executed killing effect of human lung cancer cell lines through mitochondrial dysfunction [17]. Moreover, recent studies confirmed these, which reported that NTP induced apoptosis of head and neck cancer cells by a mechanism involving MAPK-dependent mitochondrial ROS [10] and that ROS/RNS triggered JNK and p38 pathways which promoted mitochondrial perturbation and apoptosis [11].

To fundamentally understand and elucidate the effect of NTP on biological pathways, high-throughput microarrays, as an ideal screening tool for gene expression profiling analysis, could help us investigate the underlying genetic characteristic of processes involved in plasma-induced cellular or molecular responses.

The goal of the present study was to analyze the gene expression after NTP exposure that might be involved in cellular apoptosis, signaling transduction and stress response. Therefore, typical non-small cell lung cancer cells A549 were exposed to NTP with helium as the carrier gas. With the high-density microarray technique, the transcriptome for genes regulated by NTP were screened. The results showed different dose-dependent profile patterns upon various NTP exposure durations, and showed that the NTP exposure modulated the transcriptional factors (e.g., Jun, Fos and CEBPB), cytokines (e.g., IL-6, IL-8), leukocyte recruitment and activation factors (e.g., CCL20, CXCL1, CXCL2, CXCL3 and CSF2), enzymes of phosphatase (e.g., DUSP1 and DUSP2) and cyclooxygenase (e.g., PTGS2). Furthermore, several signaling pathways related to stress defensing mechanisms as well as key transcriptional processes were identified.

\section{Results}

\section{Non-thermal plasma-induced differential gene expression} in A549 cell

Using the microarray based approach, we analyzed the cellular gene expression profile of lung adenocarcinoma A549 cells upon treatment with non-thermal plasma, and focused on finding plasma-associated molecular signatures to elucidate the impact of NTP on the transcriptome of this tumor cell.

Even though the survival of the 3-min treatment group decreased to only approximate $20 \%$ at $4 \mathrm{~h}$ post exposure, when compared to the sham control (Fig. 1), the RNA integrity number (RIN) still showed that RNA was not degraded and had sufficiently high quality for further analysis (data not shown).

With the selection criteria mentioned above, 1209 differentially expressed genes were obtained for all time points. Specifically, at $4 \mathrm{~h}$ after the 1-min NTP treatment, 802 genes (559 down-regulated and 243 genes) showed significant expression according to the preset criteria (with fold changes more than 1.2, and FDR $p$ value less than 0.05), whereas only 10 genes (10 down-regulated genes), 132 genes (109 down-regulated and 23 up-regulated genes) and 773 genes (684 down-regulated and 89 up-regulated genes) expressed at 1, 2 and 4 h, respectively, after 3-min NTP exposures (Fig. 2). These data have been deposited in NCBI's Gene Expression Omnibus and are accessible through GEO Series accession number GSE59997, and the complete overview on the differentially regulated genes could be achieved by GEO2R or other software.

\section{Gene ontology and pathway enrichment analysis}

To retrieve the functional information on differentially expressed genes in each experimental group, co-regulated 

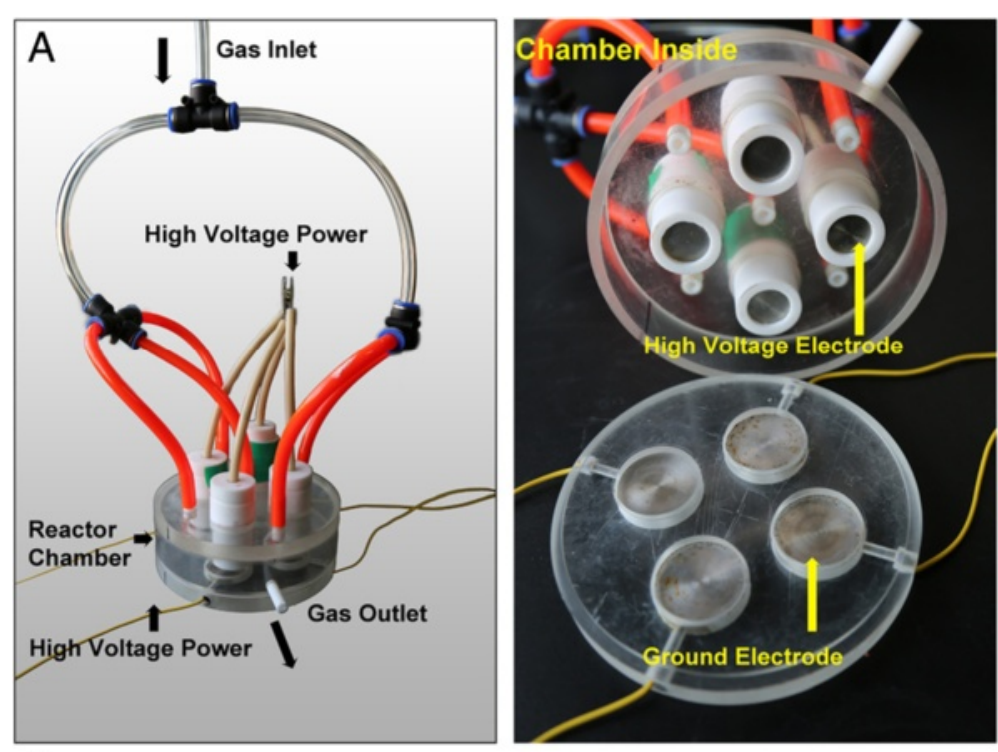

B

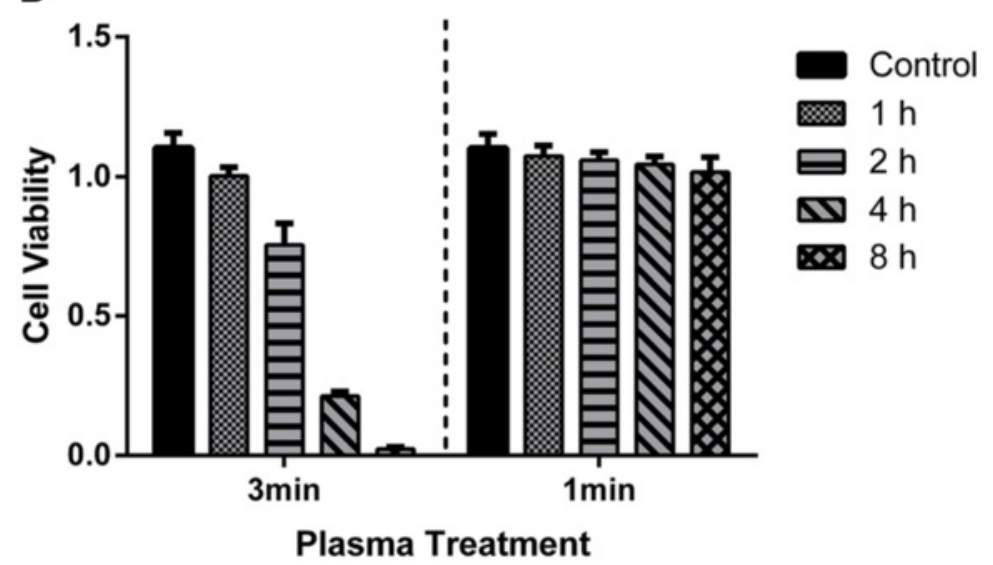

Fig. 1 The cell viability assessment of NTP on treated A549. a Photographs of a plasma jet and the plasma system. $\mathbf{b}$ The assessment of cell viability of A549 cell after NTP exposure for 1 min right panel and 3 min left panel, respectively. Time points of 1, 2, 4 or $8 \mathrm{~h}$ for viability measurements after NTP exposure were adopted. The mean and SD are shown for three independent experiments. The t-test was applied for the comparative analysis between the treatment group and the control group. The p-values were shown while ns indicated no significant difference

genes were further classified with BiNGO application with plugged-in Cytoscape. The individual expression products of the identified genes were distributed in different cellular compartments, functional classes, and involved numerous molecular and biological processes. The bar charts (Fig. 3) illustrated the affiliation of the top 20 terms of the regulated genes (FDR $p<0.05$ ) with gene class ontologies identified for each group. The whole GO terms for each time point or group could be found in the Additional file 1: Table S1.

Firstly, the GO terms of the whole transcriptome were compared with NTP to understand which categories in response to biological processes were enriched in plasmatreated cells.

At any time point after different NTP treatments, various genes were regulated and involved in a multitude of biological processes. Top hits among the biological process categories also comprised specific aspects of negative regulation of biological process, such as apoptosis and cell proliferation, response to stress and transcription factor activity, which concentrated on up-regulation of gene clusters. Meanwhile, chromosome organization and cell cycle terms were enriched in down-regulated gene clusters at $4 \mathrm{~h}$ in the 1-min NTP-exposed group (upper panel in Fig. 3). In contrary to the 1-min NTP exposure, the 3-min NTP treatment caused differential GO categories. At $2 \mathrm{~h}$ post the 3-min NTP exposure, the up-regulated genes focused on transcription regulation, and down-regulated genes clustered into nucleotide, metabolic process and methyltransferase activity (middle panel in Fig. 3). Meanwhile, at $4 \mathrm{~h}$ after exposure, the top categories comprised nucleosome/chromatin assembly, DNA behavior involved 


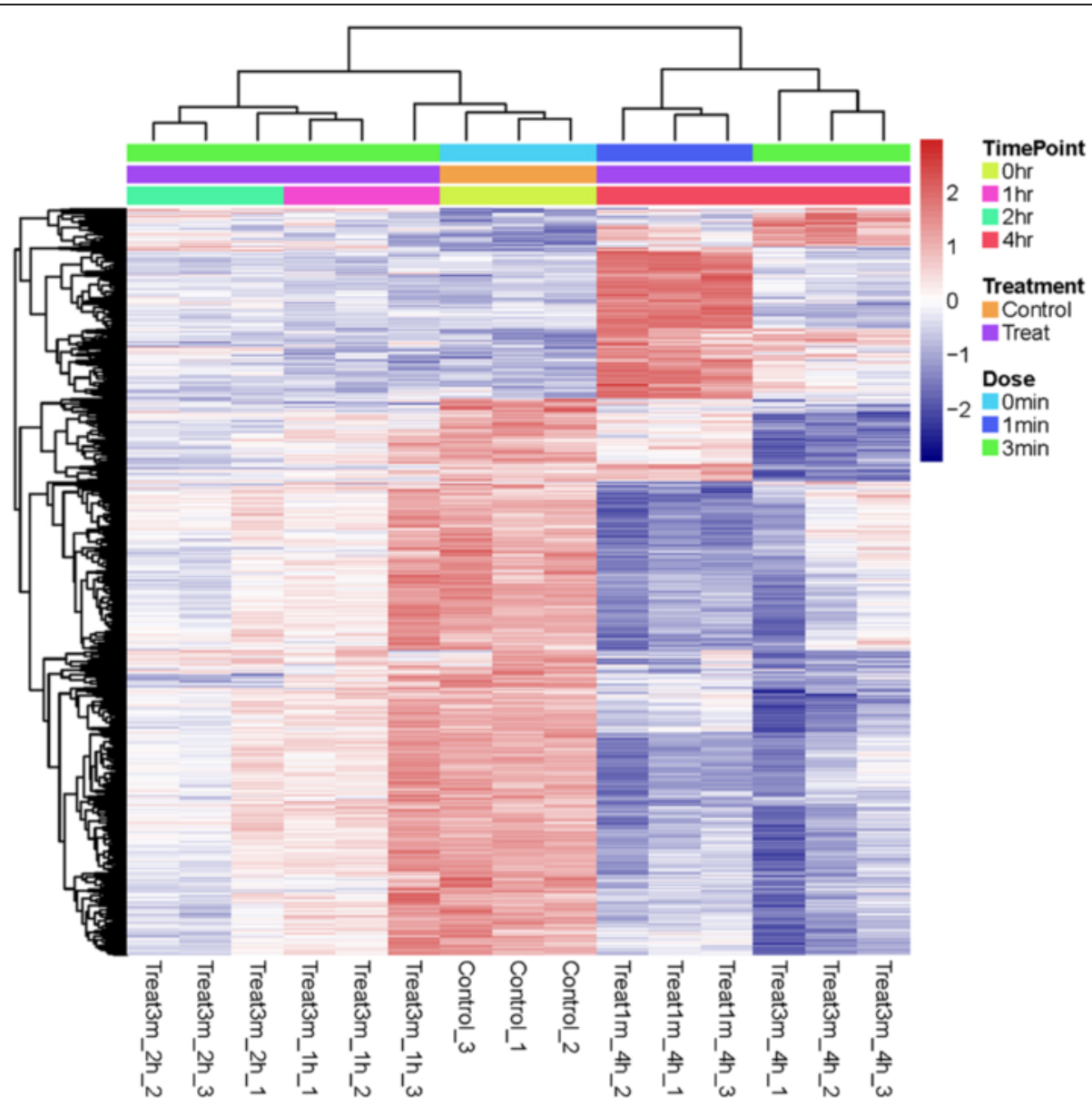

Fig. 2 The heatmap of differentially expressed genes. Patterns of changes in transcript abundance are shown on a heatmap for a robust set of 1209 transcripts using a BH p-value of 0.05 and fold changes > 1.2. Red color represents relative increase in abundance, blue color represents relative decrease, and white color represents no change. The color bars above the map indicate the time points and NTP doses.

in conformation change and DNA replication in upregulated gene list, however most of the regulations involved in metabolic processes, nucleus and molecular binding were observed in the down-regulated genes (lower panel in Fig. 3).

In the next step, we utilized gene ontology enrichment analysis to concentrate on the GO categories that were really pivotal at the indicated time point of each group. Briefly, different NTP exposure durations led to distinct time-dependent profiling patterns. Specifically, the downregulated genes after the 1-min NTP exposure were mainly related to cellular metabolism including chromosome processes, cell cycle, ATPase activity, and nucleosidetriphosphatase activity. Meanwhile, the up-regulated genes were related to kinase activity, transcription factor activity, and response to reactive oxygen species (Fig. $4 \mathrm{~A}$ ). Interestingly, the gene list for $2 \mathrm{~h}$ post 3-min NTP exposure focused on methyltransferase activity, which was a unique gene set among all groups. The enrichment gene sets at $4 \mathrm{~h}$ after the 3-min NTP exposure was mostly related to negative or down regulation of cellular processes. These genes were involved in the macromolecule metabolic process, methylation, nucleoplasm, cell cycle, and DNA metabolic process and ATP energy metabolism (Fig. 4 C). All the genes mentioned above are known to be important in the stimulation of cell proliferation and recruitment.

Furthermore, in the pathway enrichment analysis, the regulated genes for 1-min NTP exposure were clustered into biological groups which were different from those corresponding to the 3-min NTP exposure. For instance, it was found that the 1-min NTP exposure significantly repressed the expression of genes involved in mitotic telophase/cytokinesis and prometaphase as well as cell cycle, including DNA damage response and miRNA mediated regulation. Moreover, the 1-min NTP exposure also activated some of pathways including the IL-4 signaling pathway, p53 mediated signaling pathway, MAPK, TGF- $\beta$ and TNF signaling pathways (Fig. 4 B). However, the 3-min NTP exposure caused up-regulated genes like histones which played a role in HATs acetylate histones 


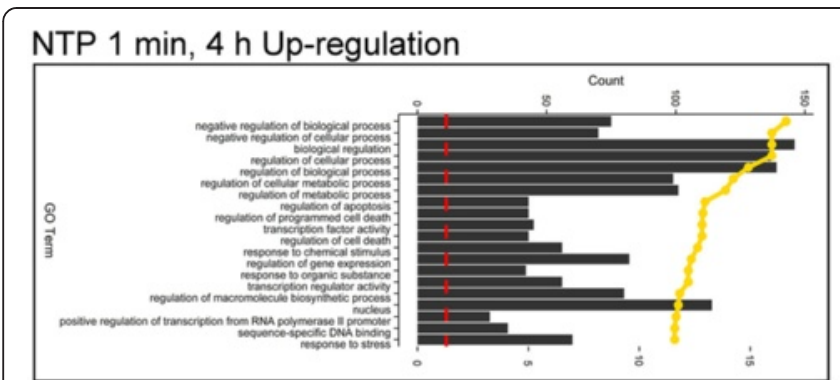

NTP 3 min, 2 h Up-regulation

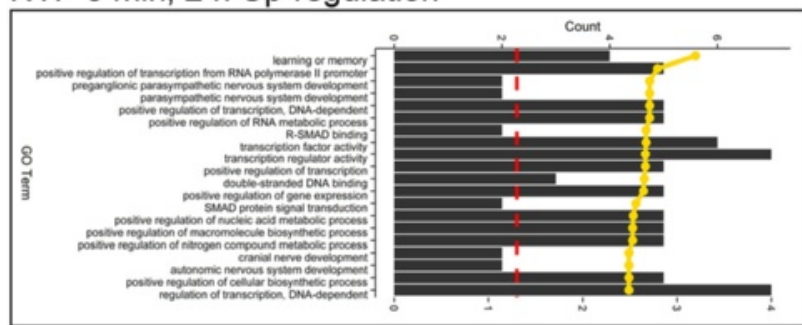

NTP 3 min, 4 h Up-regulation

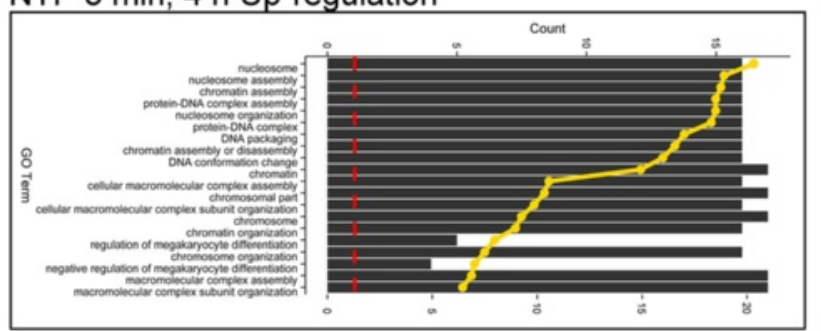

NTP $1 \mathrm{~min}, 4 \mathrm{~h}$ Down-regulation

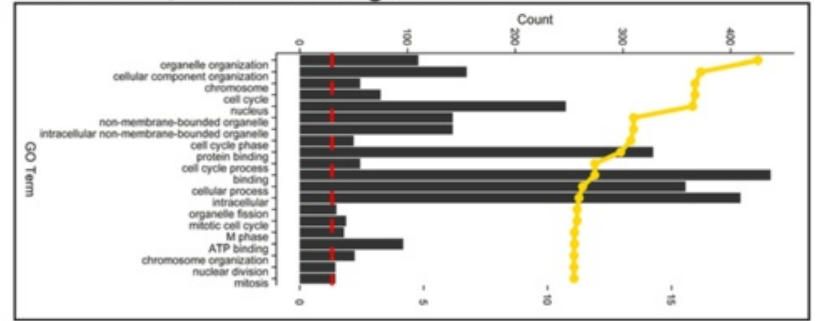

NTP $3 \mathrm{~min}, 2 \mathrm{~h}$ Down-regulation

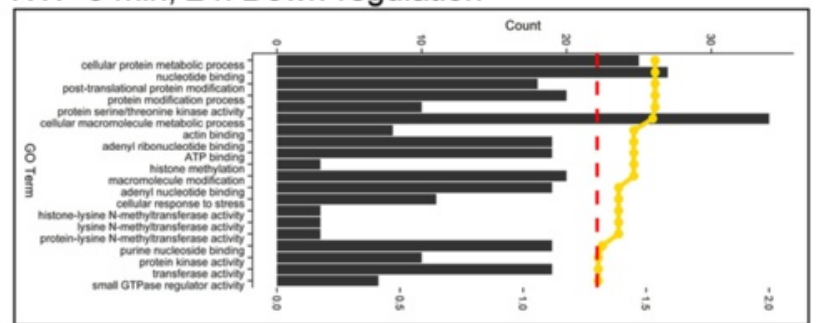

NTP $3 \mathrm{~min}, 4 \mathrm{~h}$ Down-regulation

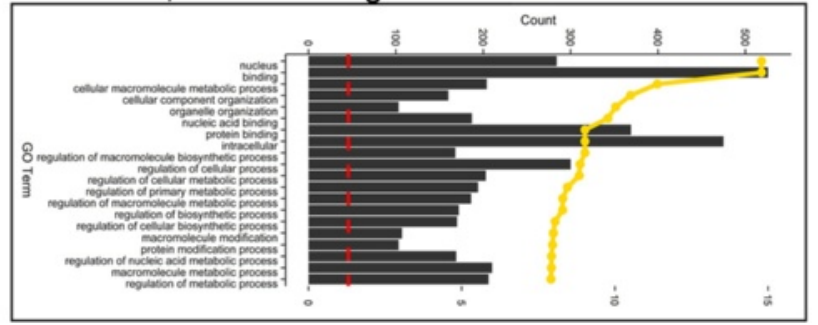

Fig. 3 The gene ontology category of each group at indicated time points. The Gene Ontology analysis was performed by BiNGO plugged-in Cytoscape. The Biological Processes (BP), Molecular Function MF and Cellular Components CC terms were involved. To include only significant results, the FDR threshold was set to 0.05 . The top 20 terms were selected, and represented as bar plots with gene number involved in these GO terms (with reference to the upper y-axis). Moreover, the corrected $p$ values were also obtained as indicated by the yellow dotted line, which had been transformed into log10 with reference to the lower $y$-axis. The red dashed line indicated the threshold of adjusted $p$ values $[-\log 10(0.05)]$

and RNA polymerase involved in transcription regulation processes. In contrast, the 1-min NTP exposure reduced the expression of genes involved in regulation of the IL-4 signaling pathway and the TGF- $\beta$ signaling pathway (Fig. 4 D). However, the effect of extended NTP processing time on gene profiles and cellular behavior and metabolism was not clear.

\section{Involved hub transcription factors}

Considering that some factors play critical roles in biological processes and interact with abundant related genes or proteins, the top hits proteins were sorted and assessed from protein-protein interaction (PPI) network. Apparently, the 1-min NTP exposure induced specific patterns that included some specifically up-regulated genes. These genes were involved in GTP and GDP energy metabolism (e.g., ARL5B and ARL14), and $\mathrm{Ca}^{2+}$ ion flow or release (e.g., ITPRIP) (Left panel of Fig. 5). Moreover, some transcription factors that played important roles in transcription and regulation were also significantly enhanced (Right panel of Fig. 5). JUN and FOS were thought to play important roles in signal transduction, cell proliferation and differentiation. CCAAT/Enhancer Binding Protein (C/EBP) (e.g., CEBPA, CEBPB) were also important transcriptional activators that regulated expression of genes involved in immune and inflammatory responses. They could bind to regulatory regions of several acute-phase and cytokines genes, and probably played a role in the regulation of acute-phase reaction and inflammation. NFKBIA inhibited the activity of NF- $\mathrm{kB} / \mathrm{REL}$ complex by trapping REL dimers in the cytoplasm through masking their nuclear localization signals. Additionally, other processes were also present, which involved tyrosine-kinase-based signaling related to cell adhesion (e.g., NEDD9), and cell cycle phase transition (e.g., GDF9).

\section{Responded multiple pathways}

Differential gene expression in response to the NTP exposure was assessed to elucidate effects of diverse pathways on the cancer cell. Pathway enrichment analysis 


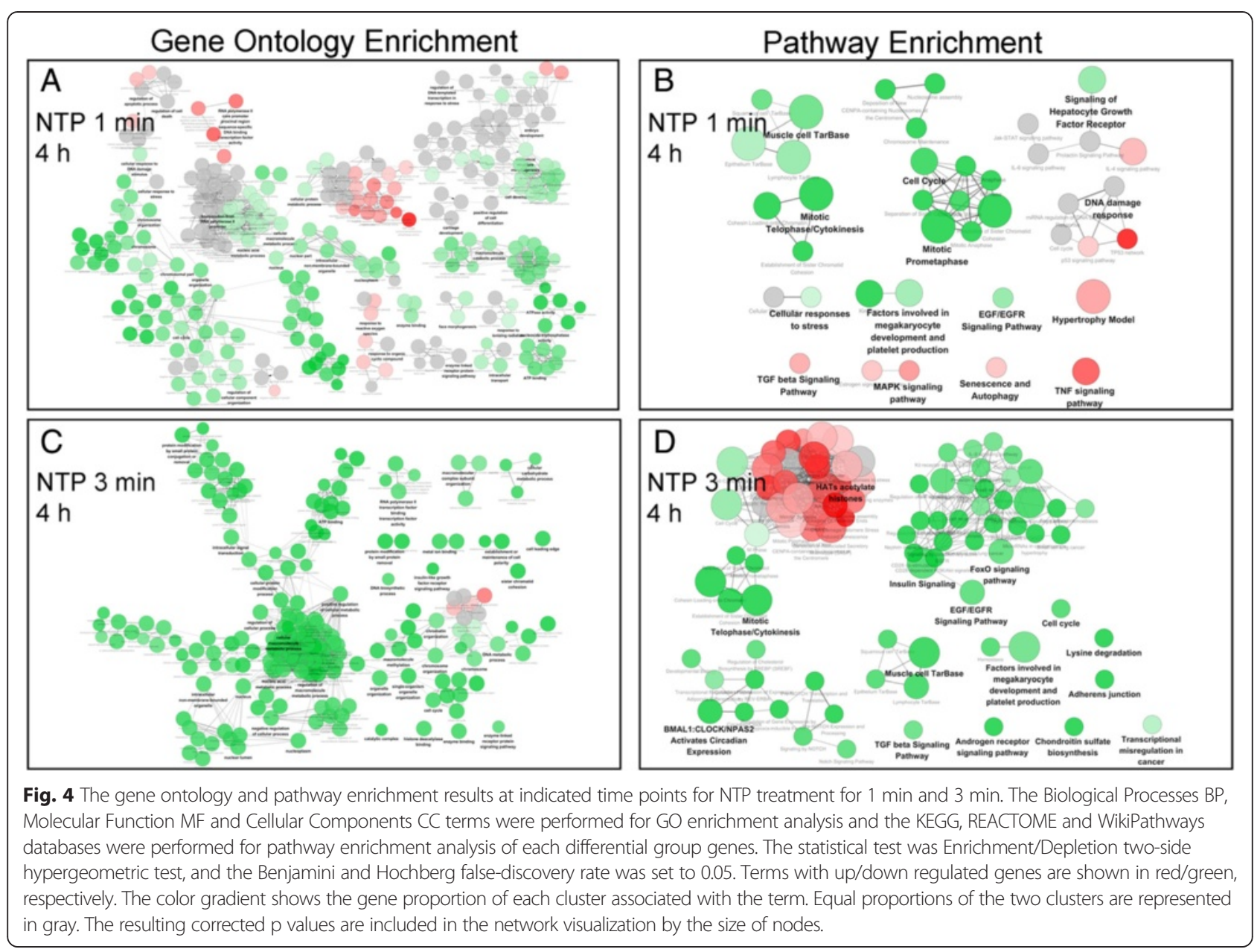

and PID top hits genes categorized several genes involved in the response to NTP exposure, which comprised transcription factors (e.g., Jun, JunB, Fos and CEBPB), negative intracellular signaling factors (e.g., NFKBIA, SOCS3 and TNFAIP3), leukocyte recruitment and activation factors (e.g., CCL20, CXCL1, CXCL2, CXCL3 and CSF2), enzymes of phosphatase (e.g., DUSP1 and DUSP2) and cyclooxygenase (e.g., PTGS2). In the pathway enrichment results, the up-regulated pathways included IL-4, TNF, TGF- $\beta$ and MAPK signaling pathways, which constituted a network with centralization of JUN, FOS and JAK critical transcription factors (Fig. 6). Among these, the transcription factors were upregulated at least 8 -fold in the 1 -min plasma-treated cells. Next, we focused on changes in expression values of genes whose transcription levels increased under stressful growth arrest conditions. The p53 was a hub of response to stress signals, as shown in the right box of Fig. 6. GADD45 was a DNA-damage inducing gene, mediating the activation of the $\mathrm{p} 38 / \mathrm{JUK}$ pathway via MTK1/MEKK4 kinase. DUSP also negatively regulated members of MAP kinase superfamily (e.g., MAPK/ERK,
SAPK/JNK and p38), which was associated with cellular proliferation and differentiation. All the genes mentioned above displayed enhanced mRNA expression.

\section{qPCR and Western blot validation}

To further validate some of the microarray profiles, seven representative candidate genes were selected for analyzing the gene expression levels via the qPCR method. The regulation patterns for all selected genes were consistent with the microarray data and only had marginal differences in the relative fold changes, except the EGR1 and FOS genes which presented extremely high mRNA expression levels by qPCR when compared to microarray analysis (shown in Table 1). Moreover, among the microarray results, we focused on and selected several candidate proteins for multiple pathways, which included apoptosis, MAPK signaling pathway, JNK, Akt and NF-kB pathways, and some critical transcriptional factors (c-Jun, JunB and c-Fos). Among the western blot results, the caspase- 3 and PARP degraded over the time points, which indicated the classical apoptosis cascade. mTOR and phosphorylated mTOR levels 


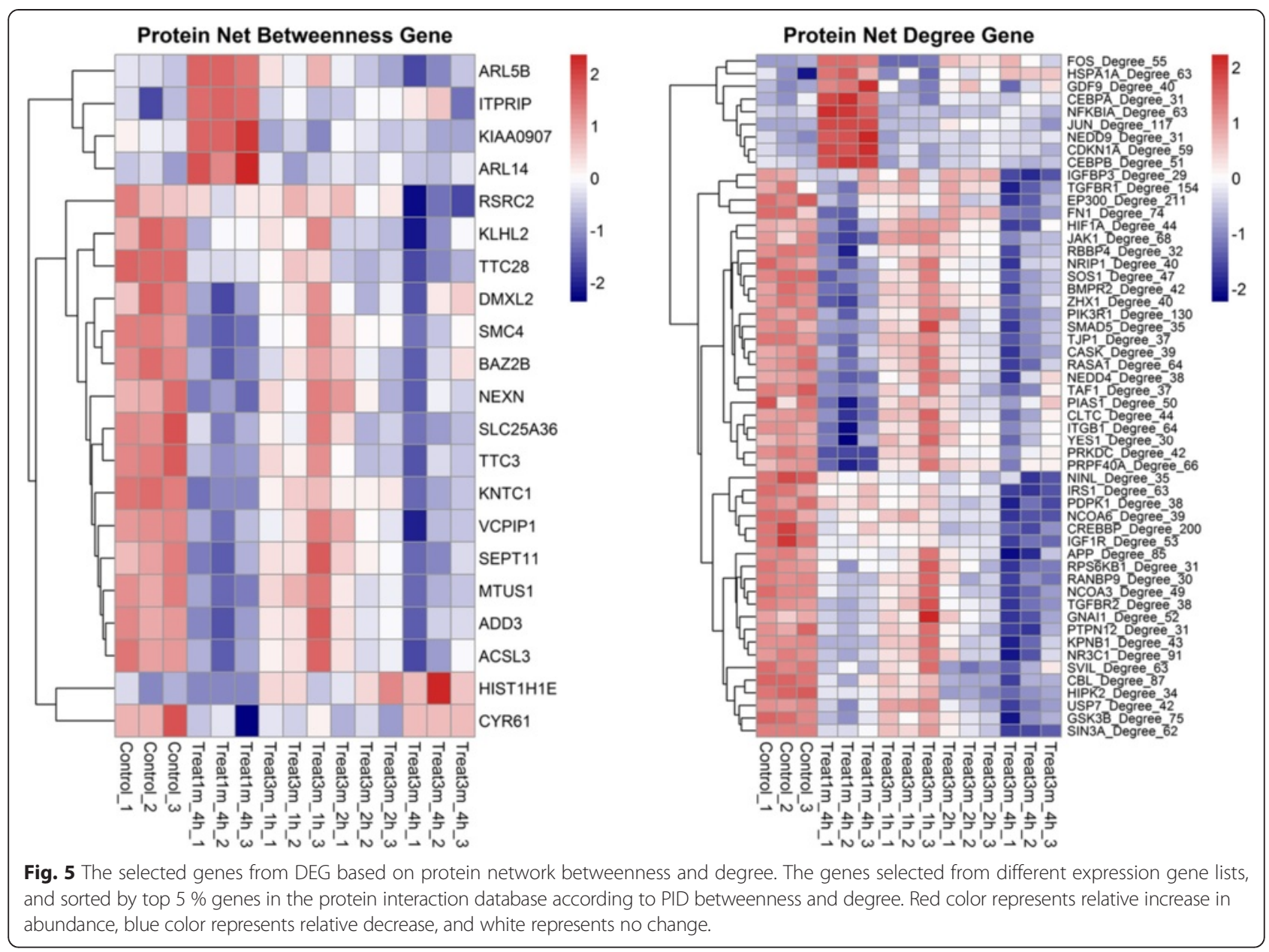

peaked at $8 \mathrm{~h}$ post NTP treatment and descended thereafter. mTOR is a serine/threonine protein kinase which is a central regulator of cellular metabolism, growth and survival in response to hormones, growth factors, nutrients, energy and stress signals [18]. It plays a critical role in the phosphorylation of AKT [19], which has also been validated in our Western blot result in that the expression of phospho-Akt was increased till $8 \mathrm{~h}$ after exposure. After that, the Akt regulated the NF- $\mathrm{kB}$ signaling pathway by inducing release of NF- $\mathrm{kB}$ through phosphorylation and degradation of IкB $\alpha$ (shown in the left panel of Fig. 7). NTP stimuli activated JNK, and phosphorylation of JNK on the site of Thr183/Tyr185 was increased in the time course, which modified the activities of numerous proteins
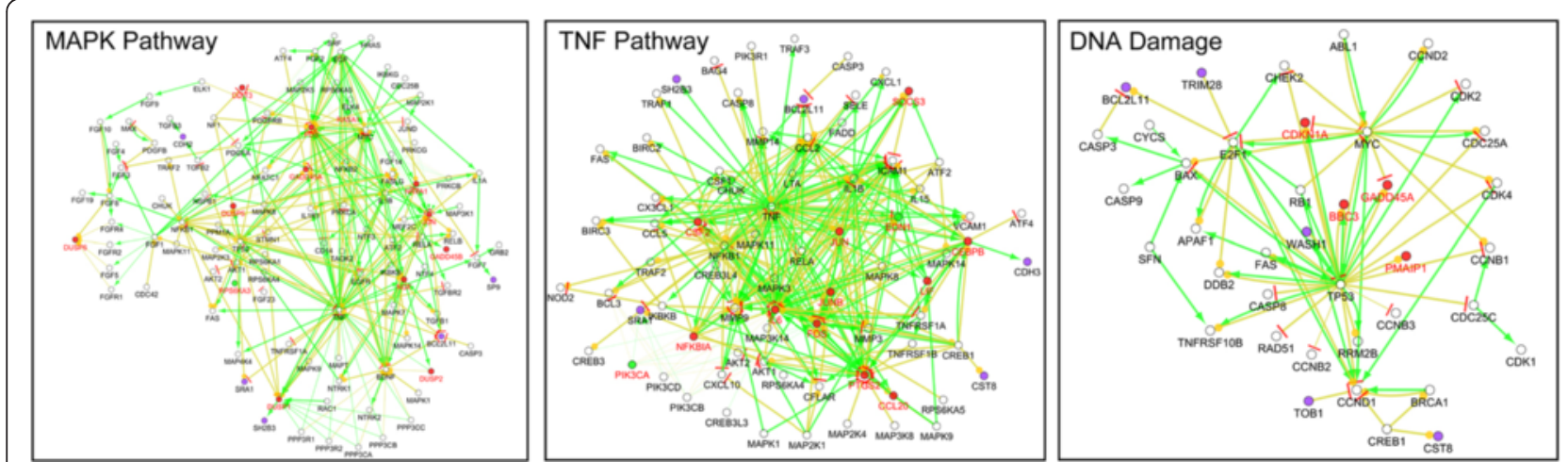

Fig. 6 The details of selected pathways after NTP exposure. The illustration showed gene correlation of indicated pathway enrichment in details by CluePedia plugged-in Cytoscape. The nodes represented genes in each pathway, with red and green colors of nodes indicating up- or down-regulated genes, respectively. The green line and yellow line represented activation and expression relationship in gene interaction enrichment, respectively. 


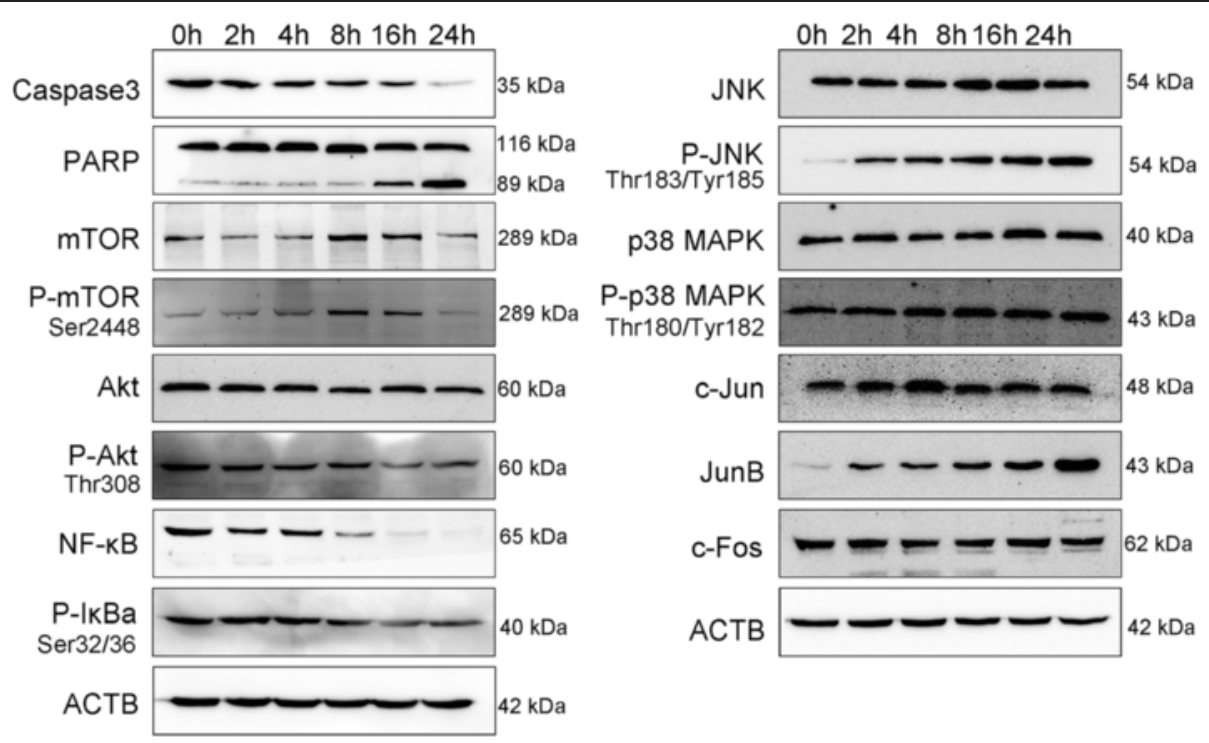

Fig. 7 Western blot analysis and validation of several key proteins from microarray results. A549 cells were collected and lysed after 1-min NTP treatment at different time points, namely, 2, 4, 8, 16 and $24 \mathrm{~h}$. With SDS-PAGE gel electrophoresis, the expressions of Caspase3, PARP, mTOR, phospho-mTOR, Akt, phospho-Akt, NF-KB, phospho-IKBa, JNK, phospho-JNK, p38 MAPK, phospho-p38 MAPK, c-Jun, JunB, c-Fos, and ACTB were measured.

that resided at the mitochondria or acted in the nucleus, and were involved in apoptosis, cell differentiation and proliferation. Moreover, the JNK activated the AP-1 transcriptional factors (c-Jun, JunB and c-Fos) [20]. Only JunB showed continued enhancement with in the time course after NTP treatment, while the expression levels of c-Jun and c-Fos remained stable. Overall, from the translational level results, NTP treatment induced cell apoptosis and cell cycle arrest to assist processes for cell repair.

\section{Discussion}

The impact of NTP on cancer cells has not been investigated using the microarray approach in detail to date. The present work was the first to employ this high-throughput technique to elucidate the responses of NSCLC tumor cells to a particular NTP treatment in vitro. The microarray technique was applied to determine the activities of thousands of genes at once to create a partial picture of cellular functions of A549 cells following NTP exposure.
In a previous study [21], the microarray technique had been used and to examine the therapeutic potential of a plasma jet on cancer cell lines and tumors, with a focus on the capability of selective tumor cell eradication and on deregulation of signaling pathways. These selective effects of NTP on different cell types can find important applications in cancer treatment [21].

Besides the apoptotic effects induced by long-time (3 min) NTP exposures to A549 cells, we also focused on the biological effects induced by short-time $(1 \mathrm{~min})$ NTP exposures, which were below the levels that induced apoptosis in most of the cells. The time points of 1,2 and $4 \mathrm{~h}$ post cell treatment were selected for detection of early effects in terms of NTP modulated gene activities, which were indeed confirmed by the microarray data.

The expressions of more than 1200 genes were modulated by NTP exposures, with at least 1.5-fold induction or repression when compared to those of non-treated

Table 1 Verification of gene expression changes in 1-min NTP Treatment at $4 \mathrm{~h}$ after exposure

\begin{tabular}{llll}
\hline Gene Symbol & qPCR relative change & Microarray relative change (log fold change) & $p$-value \\
\hline CXCL2 & $3.115 \pm 1.007$ & $1.802 \pm 0.029 * *$ & 0.0016 \\
CXCL3 & $2.885 \pm 0.296$ & $1.786 \pm 0.040 *$ & 0.0364 \\
EGR1 & $33.712 \pm 11.183$ & $5.437 \pm 0.129 * * *$ & 0.0003 \\
FOS & $48.040 \pm 18.817$ & $4.650 \pm 0.058 * * * *$ & $<0.0001$ \\
JUN & $9.808 \pm 0.892$ & $3.471 \pm 0.003 * * * *$ & $<0.0001$ \\
JUNB & $4.554 \pm 0.977$ & $1.945 \pm 0.238$ & 0.1118 \\
TNFAIP3 & $3.194 \pm 0.212$ & $2.313 \pm 0.019 *$ & 0.0153 \\
\hline
\end{tabular}

Note: The results were shown as mean $\pm S D$ in the $2^{\text {nd }}$ and 3 ed columns, and asterisks indicated significant differences between two methods found using t-test 
cells. Our results showed that the affected genes differed significantly between the control and treated cells depending on the conditions. Both sub-groups (e.g., up- and down-regulated genes) contained differentially expressed genes encoding for molecules known to participate in the apoptotic process, cellular response to stress, chromosome organization, cell cycle, DNA damage response and miRNA regulation. The signaling and network analysis by ClueGO/CluePedia revealed that certain significantly regulated genes were involved in pathways which led to the p53 signaling pathway, MAPK signaling pathway, TGF and TNF signaling pathways, and IL-4/IL-6 signaling pathways. Likewise, the majority of the biological processes that decreased the expressions were mainly related to chromosome organization/assembly, cell cycle and metabolic processes.

The most striking effect of NTP in the gene expression profiling analysis was the modulation of a large set of genes that were involved in multiple signaling pathways, including PI3K/Akt and NF- $\mathrm{KB}$ mediated TNF signaling, MAPK, Jak-STAT, TGF- $\beta$ and VEGF pathways. To better understand the influence of plasma-induced reactive species and potentially beneficial mechanisms of NTP on A549 cells, we examined the protein expression of apoptosis and related transcriptional factors which were mostly up-regulated or down-regulated after NTP exposure. A number of transcripts were identified to be significantly modulated for transcriptional factors. In this regard, ClueGO predicted the involvement of FOS/JUN/ p53 in intracellular signaling in our experimental setup.

Further analysis of our data suggested that NTP might activate a broader range of p53-relevant processes. It is known that p53 is a critical transcription factor which participates in transcription as well as signal transduction, including cell cycle arrest [22], apoptosis [23, 24], DNA repair [25] and damage prevention, cellular senescence and p53 negative feedback. The Western blot assay also confirmed the multiple pathways involved in these complicated processes. The MAPK, JNK and NF$\kappa \mathrm{B}$ pathways were all involved, and played critical roles in the regulation of responses to external stresses.

In summary, the large number of negatively regulated genes involved in biological or cellular processes reflected the cellular responses to NTP, and helped confirm specific pathways or stress responses to NTP. Although the details regarding the activation of specific pathways remained to be elucidated, our data presented new evidence that NTP treatment of A549 cells modulated the transcription of a variety of genes, which might center on p53 and MAPK and TNF signaling pathways, etc. Our data further indicated that it was possible to modulate cellular signaling as well as metabolism and/or apoptosis processes in NTP treated tumor cells on a genetic level.

\section{Conclusions}

Overall, non-thermal plasma treated cells exhibited a pattern typical of damage responses, thereby validating several critical pathways/regulators, presumably mediated through multiplex signaling pathways. Further investigation of these genes in other cell lines may reveal comprehensive mechanisms of plasma induced effects.

\section{Methods}

\section{Ethics statement}

All procedures involving human cell line use were approved by the Hefei Institutes of Physical Science Committee (Chinese Academy of Sciences), where the approval number was 2014021.

\section{Cell culture}

The human lung adenocarcinoma epithelial cell line A549, purchased from ADCC and stored in our laboratory, were cultured in DMEM (Hyclone, Logan, US) supplemented with $10 \%$ fetal bovine serum (FBS) (Hyclone, Logan, US), $1 \%$ penicillin/streptomycin and $2 \mathrm{mM} \mathrm{L-}$ glutamine. All cultures were grown in a humidified $5 \%$ $\mathrm{CO}_{2}$ atmosphere at $37^{\circ} \mathrm{C}$.

\section{Non-thermal plasma equipment}

The atmospheric pressure dielectric barrier discharge (DBD) plasma is schematically illustrated in Fig. 1 A, which refers to the setup described elsewhere in previous researches $[1,26]$. The four DBD plasma reactors are sealed in a hollow plexiglass cylinder as a reactor chamber with two orifices. One is for injection of working gas, and the other is for gas exhaust from the chamber. The high-voltage electrode is a 32-mm-diameter copper cylinder, which is covered by a 1-mm-thick quartz glass as an insulating dielectric barrier. The ground electrode is a 37-mm-diameter copper cylinder. The discharge gap between the bottom of the quartz and the treated sample surface is fixed at $5 \mathrm{~mm}$. The alternating current power supply is a commercial transformer capable of generating continuous and tunable output voltages and frequencies. The applied voltage and discharge current of the DBD plasma are monitored on a Tektronix MSO 5104 digital oscilloscope equipped with a high voltage probe (Tektronix P6015A) and current probe (Tektronix P6021). The gas flow into the chamber from the gas inlets had a fixed flow rate of $80 \mathrm{~L} / \mathrm{h}$. In order to expel air as much as possible from the reactor chamber, helium was injected 5 min before the experiment. The non-thermal plasma is generated by a voltage of $12 \mathrm{kV}$ (peak to peak) with a frequency of $24 \mathrm{kHz}$. The discharge power density was measured to be about $0.9 \mathrm{~W} / \mathrm{cm} 3$. The typical optical spectrum of the helium DBD plasma was shown in Additional file 2: Figure 
S1, while the typical current and voltage of the helium DBD discharge were shown in Additional file 3: Figure S2.

\section{Cell viability assay}

The viability of cells was assessed with the Cell Counting Kit-8 (Beyotime, China) following the manufacturer's instructions. At $24 \mathrm{~h}$ after NTP exposure, the cells were treated with CCK-8 reagent for $1 \mathrm{~h}$ at $37{ }^{\circ} \mathrm{C}$, and then $200 \mu \mathrm{l}$ of the supernatant was transferred into the 96well plates. The absorbance was measured at $450 \mathrm{~nm}$ with a Varioskan Flash microplate reader (Thermo Fisher Scientific, Rockford, IL, USA)

\section{NTP treatment and RNA preparation}

A549 cells were passaged $24 \mathrm{~h}$ before NTP treatment. Cells $\left(3 \times 10^{5}\right)$ were seeded into $35 \mathrm{~mm}$ dishes and were allowed to grow to $80 \%$ confluence before NTP exposure. Cells with $3 \mathrm{ml}$ culture medium were treated directly with NTP. After 1-min or 3-min exposures, the cells were returned into the incubator for further culture for a specific duration, and then harvested by trypsinization and centrifugation. The cell pellets were lysed with TRIzol (Life technologies, Carlsbad, CA, US) and stored at $-80{ }^{\circ} \mathrm{C}$ for further studies.

The total RNA was extracted with TRIzol Reagent (Life technologies, Carlsbad, CA, US) following the manufacturer's instructions, and checked for an RNA integrity number (RIN) to inspect the RNA integrity with an Agilent Bioanalyzer 2100 (Agilent technologies, Santa Clara, CA, US).

Qualified total RNA was further purified with RNeasy micro kit (QIAGEN, GmBH, Germany), and genomic contamination was removed with RNase-Free DNase Set (QIAGEN, GmBH, Germany). The purified RNA was stored at $-80{ }^{\circ} \mathrm{C}$.

\section{Microarray hybridization}

The total RNA was amplified, labeled and purified using the GeneChip 3'IVT Express Kit (Affymetrix, Santa Clara, CA, US) following the manufacturer's instructions to obtain biotin-labeled cRNA. After hybridization on Human PrimeView Arrays for $16 \mathrm{~h}$ at $45^{\circ} \mathrm{C}$ and $60 \mathrm{rpm}$ in Hybridization Oven 640 (Affymetrix, Santa Clara, CA, US), slides were washed and stained with a Fluidics Station 450 (Affymetrix, Santa Clara, CA, US). Scanning was performed on a seventh-generation GeneChip Scanner 3000 (Affymetrix, Santa Clara, CA, US). The Affymetrix GCOS software was used to perform image analysis and to generate raw intensity data. These microarray data have been deposited in NCBI's Gene Expression Omnibus and are accessible through GEO Series accession number GSE59997.

\section{Microarray data analysis for Differential Expression Gene (DEG)}

All microarray data analyses were performed in $\mathrm{R}$ (http://www.r-project.org/) with multiple packages involving the Affymtriex platform for data analysis and statistical analysis in the subsequent Bioconductor. The initial data quality was assessed by the background level, labeling bias, and pair-wise correlation among samples.

For PrimeView Chip, the customized CDF file (version 17, ENTREZG) downloaded from BrainArray website was performed in probe set mapping [27, 28]. IQR was used for raw data filtering with the genefilter package, with the threshold set to remove the intensity less than $20 \%$ of IQR global intensity. Normalization was performed with RMA algorithm which included the global background adjustment and quantile normalization. Empirical Bayes moderation of the standard error and Benjamini and Hochberg false-discovery rate correction for multiple testing were employed, again as implemented in the limma package [29]. Differentially expressed genes were identified with threshold fold changes of more than 1.2 and $\mathrm{BH}$ adjusted $p$ value less than 0.05 .

\section{Gene ontology categories analysis}

To link our data to prior knowledge, we performed GOEA by using the Cytoscape (http://www.cytoscape.org/) plug-in BiNGO (v2.44) [30]. To include only significant results, the FDR threshold was set to 0.05 . The bar plot represented gene number and the adjusted $p$ value of each gene set were used to visualize the GO categories.

\section{Pathway enrichment analysis}

We performed pathway enrichment analysis by using the Cytoscape plug-in ClueGO (v2.1.1) and CluePedia (v1.1.1)[31, 32]. The KEGG, REACTOME, and WikiPathways database had been used to enrich pathways. The Benjamini and Hochberg false-discovery rate was set to 0.05 . For KEGG pathway based data integration and visualization, the pathview package [33] was used to present some specific KEGG pathways in selected and interested pathways.

\section{Top hits genes of DEG in protein interaction network}

HPRD (Release 9) [34] was used to analyze the betweenness centrality and degree of differential expression genes in the network, which had 9,673 nodes and 39,204 PPIs. Among the exclusive experimentally derived protein-protein interaction databases, HPRD was the most complete and well overlapped with other PPI databases [35]. The data were obtained from the GCC (HPRD: 9,270 nodes and 38,855 interactions) by moving the small clusters and single nodes. All the topological parameters were computed based on the GCC [36].After 
that, the differentially expressed genes were matched to the arranged PPI datasets, and the top hits genes were identified using the characteristics of betweeness centrality and degree in PPI. The selected genes were visualized in a graphic heatmap.

\section{qRT-PCR analysis of mRNA expression}

To validate the differentially expressed mRNAs by realtime PCR, $1 \mu \mathrm{g}$ of the total RNA was reverse-transcribed with the One Step PrimeScript RT-PCR Kit (TaKaRa, Japan). Quantitative RT-PCR was carried out with a Light Cycler 480 II instrument (Roche, Indianapolis, IN). The primer sequences used for RT-PCR were available on request. Fold changes in the gene expression were calculated with the $\Delta \Delta \mathrm{Ct}$ method.

\section{Western blot}

In the present experiments, A549 cells were exposed to NTP for 1 min and harvested at 1, 2, 4 and 8 h post exposure. The cells were rinsed once with ice-cold PBS, spun down at $230 \mathrm{~g}$ for $5 \mathrm{~min}$ and lysed in ice-cold lysis buffer containing protease and phosphatase inhibitors (Thermo Fisher Scientific, Rockford, IL, USA), and then $2 \mathrm{mM}$ phenylmethanesulfonyl fluoride (PMSF) was added. Subsequently, the cells were kept on ice for $30 \mathrm{~min}$ and agitated every $10 \mathrm{~min}$ to ensure a complete cell lysis. Lysates were centrifuged at $10^{4} \mathrm{~g}$, the supernatants were isolated, and the protein concentrations were adjusted in all samples prior to heating at $95{ }^{\circ} \mathrm{C}$ for 5 min in $1 \times$ loading buffer $(0.25 \mathrm{M}$ Tris, $2 \%$ SDS, $10 \%$ glycerol, $2 \% \beta$-mercaptoethanol, $0.004 \%$ bromphenolblue) and then subjected to SDS-PAGE (sodium dodecyl sulfate poly-acrylamide gel electrophoresis) on precast $12 \%$ PAGE gels. Proteins were blotted onto PVDF membranes. Subsequently, unspecific binding was blocked with $0.5 \%$ nonfat milk powder in Tris-buffered saline (20 mM Tris, $13.7 \mathrm{mM} \mathrm{NaCl}$ ) containing $0.1 \%$ Tween (TBS-T) for $30 \mathrm{~min}$. After this, the membrane was incubated with the corresponding primary antibody Caspase-3(\#9665), PARP (\#9532), c-Fos (\#2250), c-Jun (\#9165), JunB (\#3753), Phospho-Akt (\#2965), PhosphoІкB $\alpha$ (\#9246), p38 MAPK (\#8690), Phospho-p38 MAPK (\#4511), SAPK/JNK (\#9258), Phospho-SAPK/JNK (\#4668), NF-kB p65 (\#4764), mTOR (\#2983), PhosphomTOR (\#5536) (Cell Signaling, Beverly, MA, USA) with appropriate dilution at $4{ }^{\circ} \mathrm{C}$ overnight. This was followed by washing three times with TBS-T and incubation with horseradish peroxidase-coupled secondary antibodies $\left(1: 10^{4}\right)$ for $1 \mathrm{~h}$ at room temperature. After washing three times with TBS-T, the membranes were visualized by using the Western Blotting Substrate system. The same membranes were stripped and relabeled with antibodies directed against the corresponding total protein as well as ACTB as a loading control.

\section{Statistical analysis}

The obtained cell survival data came from three independent experiments. The Western Blot data were typical results. The results were illustrated with Prism 6.0 (GraphPad software, La Jolla, CA, USA). The mean values were plotted as bar charts with error bars, which represented standard deviations. One-way analysis of variances (ANOVA) or t-test was used to determine the statistical significance of the differences between the interested samples and the sham control.

\section{Availability of supporting data}

These microarray data have been deposited in NCBI's Gene Expression Omnibus and are accessible through GEO Series accession number GSE59997 (http://www.ncbi.nlm.nih.gov/geo/query/acc.cgi?acc=GSE59997).

\section{Additional files}

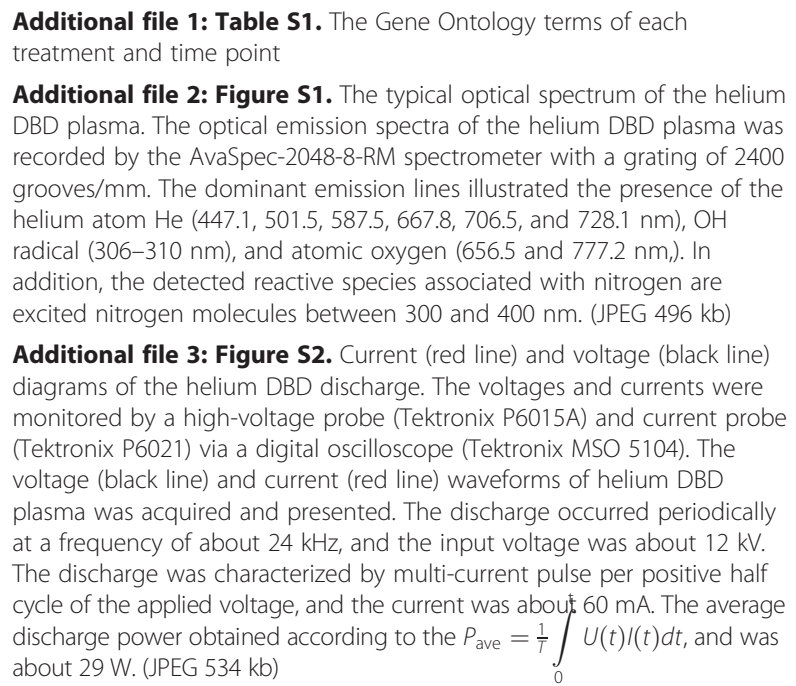

Additional file 2: Figure S1. The typical optical spectrum of the helium DBD plasma. The optical emission spectra of the helium DBD plasma was recorded by the AvaSpec-2048-8-RM spectrometer with a grating of 2400 grooves $/ \mathrm{mm}$. The dominant emission lines illustrated the presence of the helium atom $\mathrm{He}(447.1,501.5,587.5,667.8,706.5$, and $728.1 \mathrm{~nm}), \mathrm{OH}$ radical (306-310 nm), and atomic oxygen (656.5 and $777.2 \mathrm{~nm}$,$) . In$ addition, the detected reactive species associated with nitrogen are excited nitrogen molecules between 300 and 400 nm. (JPEG 496 kb)

Additional file 3: Figure S2. Current (red line) and voltage (black line) diagrams of the helium DBD discharge. The voltages and currents were monitored by a high-voltage probe (Tektronix P6015A) and current probe (Tektronix P6021) via a digital oscilloscope (Tektronix MSO 5104). The voltage (black line) and current (red line) waveforms of helium DBD plasma was acquired and presented. The discharge occurred periodically at a frequency of about $24 \mathrm{kHz}$, and the input voltage was about $12 \mathrm{kV}$. The discharge was characterized by multi-current pulse per positive half cycle of the applied voltage, and the current was about $60 \mathrm{~mA}$. The average discharge power obtained according to the $P_{\text {ave }}=\frac{1}{T} \int U(t) /(t) d t$, and was about 29 W. (JPEG 534 kb)

\section{Abbreviations}

$\mathrm{BH}$ : Benjamini and Hochberg adjust; CCL: Chemokine (C-C Motif) Ligand; C/EBP: CCAAT/Enhancer Binding Protein; CSF1: Colony Stimulating Factor 1; CXCL: Chemokine (C-X-C Motif) Ligand; DBD: Dielectric Barrier Discharge; DMEM: Dulbecco's Modified Eagle's Medium; DUSP1/2: Dual Specificity Phosphatase1/2; FBS: Fetal Bovine Serum; FDR: False Discovery Rate; FITC: Fluorescein Isothiocyanate; FOS: FBJ Murine Osteosarcoma Viral Oncogene Homolog; GEO: Gene Expression Omnibus; GO: Gene Ontology; GOEA: Gene Ontology Categories Analysis; IL6: Interleukin 6; JNK: c-JUN $\mathrm{N}$-terminal Kinase; JUN: Jun Proto-Oncogene; MAPK: Mitogen-Activated Protein Kinase; NSCLC: Non-Small Cell Lung Cancer; NTP: Nonthermal Plasma; PI: Propidium lodide; PTGS2: Prostaglandin-Endoperoxide Synthase 2; RIN: RNA Integrity Number; TGF- $\beta$ : Transforming Growth Factor Beta.

\section{Competing interests}

The authors declare that they have no competing interests.

\section{Authors' contributions}

$\mathrm{JH}$ carried out the microarray study and bioinformatics analysis and participated in the design of the study. JM carried out the western Blot study. KNY revised the manuscript. WL participated in the GPCR assays. CC designed and constructed the non-thermal plasma equipment. LZB participated in the 
cell culture viability array and the design of the study. WH participated in the design of the study. All authors read and approved the final manuscript.

\section{Acknowledgements}

The authors would like to particularly thank Dr. Zhenpeng Li for the PPI network construction and Dr. Guodong Chen for his helpful comments. This work was funded by "Hundred Talents Program" of the Chinese Academy of Sciences and Chinese Academy of Sciences (CASHIPS) Dean Fund No. YZJJ201331.

\section{Author details}

${ }^{1}$ Center of Medical Physics and Technology, Hefei Institutes of Physical Science, Chinese Academy of Sciences, Hefei, China. ${ }^{2}$ School of Life Sciences, University of Science and Technology of China, Hefei, China. Institute of Plasma Physics, Hefei Institutes of Physical Sciences, Chinese Academy of Sciences, Hefei, China. ${ }^{4}$ Department of Physics and Materials Science, City University of Hong Kong, Tat Chee Avenue, Kowloon Tong, Hong Kong. ${ }^{5}$ Mailbox 1110350 Shushanhu Road, Hefei, Anhui 230031, P. R. China.

\section{Received: 3 December 2014 Accepted: 20 May 2015}

\section{Published online: 06 June 2015}

\section{References}

1. Kalghatgi S, Kelly CM, Cerchar E, Torabi B, Alekseev O, Fridman A, et al. Effects of non-thermal plasma on mammalian cells. PLoS One. 2011;6(1):e16270.

2. Robert E, Vandamme M, Brullé L, Lerondel S, Le Pape A, Sarron V, et al. Perspectives of endoscopic plasma applications. Clin Plasma Med. 2013;1(2):8-16.

3. Zuo X, Wei Y, Wei Chen L, Dong Meng Y, Team PM. Non-equilibrium atmospheric pressure microplasma jet: An approach to endoscopic therapies. Phys Plasmas. 2013;20(8):083507.

4. Cheng X, Sherman J, Murphy W, Ratovitski E, Canady J, Keidar M. The effect of tuning cold plasma composition on glioblastoma cell viability. PLoS One. 2014;9(5):e98652.

5. Vandamme M, Robert E, Lerondel S, Sarron V, Ries D, Dozias S, et al. ROS implication in a new antitumor strategy based on non-thermal plasma. Int J Cancer. 2012;130(9):2185-94.

6. Kaushik NK, Kaushik N, Park D, Choi EH. Altered antioxidant system stimulates dielectric barrier discharge plasma-induced cell death for solid tumor cell treatment. PLoS One. 2014;9(7):e103349.

7. Kaushik N, Kaushik NK, Kim CH, Choi EH. Oxidative stress and cell death induced in U-937 human monocytic cancer cell line by non-thermal atmospheric air plasma soft jet. Sci Adv Mater. 2014;6(8):1740-51.

8. Kaushik N, Kumar N, Kim CH, Kaushik NK, Choi EH. Dielectric barrier discharge plasma efficiently delivers an apoptotic response in human monocytic lymphoma. Plasma Process Polym. 2014;11(12):1175-87.

9. Sensenig R, Kalghatgi S, Cerchar E, Fridman G, Shereshevsky A, Torabi B, et al. Non-thermal plasma induces apoptosis in melanoma cells via production of intracellular reactive oxygen species. Ann Biomed Eng. 2011;39(2):674-87.

10. Kang SU, Cho JH, Chang JW, Shin YS, Kim KI, Park JK, et al. Nonthermal plasma induces head and neck cancer cell death: the potential involvement of mitogen-activated protein kinase-dependent mitochondrial reactive oxygen species. Cell Death Dis. 2014;5:e1056.

11. Ahn HJ, Kim Kl, Hoan NN, Kim CH, Moon E, Choi KS, et al. Targeting cancer cells with reactive oxygen and nitrogen species generated by atmosphericpressure air plasma. PLoS One. 2014;9(1):e86173.

12. Kim CH, Kwon S, Bahn JH, Lee K, Jun SI, Rack PD, et al. Effects of atmospheric nonthermal plasma on invasion of colorectal cancer cells. Appl Phys Lett. 2010;96(24):243701.

13. Partecke LI, Evert K, Haugk J, Doering F, Normann L, Diedrich S, et al. Tissue tolerable plasma (TTP) induces apoptosis in pancreatic cancer cells in vitro and in vivo. BMC Cancer. 2012;12:473.

14. Kim CH, Bahn JH, Lee SH, Kim GY, Jun SI, Lee K, et al. Induction of cell growth arrest by atmospheric non-thermal plasma in colorectal cancer cells. J Biotechnol. 2010;150(4):530-8.

15. Yan X, Xiong Z, Zou F, Zhao S, Lu X, Yang G, et al. Plasma-induced death of HepG2 cancer cells: intracellular effects of reactive species. Plasma Process Polym. 2012;9(1):59-66.

16. Yan D, Sherman JH, Cheng X, Ratovitski E, Canady J, Keidar M. Controlling plasma stimulated media in cancer treatment application. Appl Phys Lett. 2014; 105(22):224101.
17. Panngom K, Baik KY, Nam MK, Han JH, Rhim H, Choi EH. Preferential killing of human lung cancer cell lines with mitochondrial dysfunction by nonthermal dielectric barrier discharge plasma. Cell Death Dis. 2013;4:e642.

18. Hay N, Sonenberg N. Upstream and downstream of mTOR. Genes Dev. 2004;18(16):1926-45.

19. Frias MA, Thoreen CC, Jaffe JD, Schroder W, Sculley T, Carr SA, et al. mSin1 is necessary for Akt/PKB phosphorylation, and its isoforms define three distinct mTORC2s. Curr Biol. 2006;16(18):1865-70.

20. Whitmarsh AJ, Davis RJ. Transcription factor AP-1 regulation by mitogenactivated protein kinase signal transduction pathways. J Mol Med. 1996;74(10):589-607.

21. Keidar M, Walk R, Shashurin A, Srinivasan P, Sandler A, Dasgupta S, et al. Cold plasma selectivity and the possibility of a paradigm shift in cancer therapy. Br J Cancer. 2011;105(9):1295-301.

22. Chen F, Chang D, Goh M, Klibanov SA, Ljungman M. Role of p53 in cell cycle regulation and apoptosis following exposure to proteasome inhibitors. Cell Growth Differ. 2000;11(5):239-46.

23. Kastan MB, Canman CE, Leonard CJ. P53, cell cycle control and apoptosis: implications for cancer. Cancer Metastasis Rev. 1995;14(1):3-15.

24. Leonard CJ, Canman CE, Kastan MB. The role of p53 in cell-cycle control and apoptosis: implications for cancer. Important Adv Oncol. 1995;33-42.

25. Pellegata NS, Antoniono RJ, Redpath JL, Stanbridge EJ. DNA damage and p53-mediated cell cycle arrest: a reevaluation. Proc Natl Acad Sci U S A. 1996;93(26):15209-14.

26. Haertel B, Volkmann F, von Woedtke T, Lindequist U. Differential sensitivity of lymphocyte subpopulations to non-thermal atmospheric-pressure plasma. Immunobiology. 2012;217(6):628-33.

27. Sandberg R, Larsson O. Improved precision and accuracy for microarrays using updated probe set definitions. BMC Bioinformatics. 2007;8(1):48.

28. Dai M, Wang P, Boyd A, Kostov G, Athey B, Jones E, et al. Evolving gene/ transcript definitions significantly alter the interpretation of GeneChip data. Nucleic Acids Res. 2005;33:e175.

29. Smyth GK. Linear models and empirical bayes methods for assessing differential expression in microarray experiments. Stat Appl Genet Mol Biol. 2004;3:Article3.

30. Maere S, Heymans K, Kuiper M. BiNGO: a Cytoscape plugin to assess overrepresentation of gene ontology categories in biological networks. Bioinformatics. 2005;21(16):3448-9.

31. Bindea G, Galon J, Mlecnik B. CluePedia Cytoscape plugin: pathway insights using integrated experimental and in silico data. Bioinformatics. 2013;29(5):661-3.

32. Bindea G, Mlecnik B, Hackl H, Charoentong P, Tosolini M, Kirilovsky A, et al. ClueGO: a Cytoscape plug-in to decipher functionally grouped gene ontology and pathway annotation networks. Bioinformatics. 2009;25(8):1091-3.

33. Luo W, Brouwer C. Pathview: an R/Bioconductor package for pathway-based data integration and visualization. Bioinformatics. 2013;29(14):1830-1.

34. Keshava Prasad TS, Goel R, Kandasamy K, Keerthikumar S, Kumar S, Mathivanan S, et al. Human Protein Reference Database-2009 update. Nucleic Acids Res. 2009;37(Database issue):D767-72.

35. Mathivanan S, Periaswamy B, Gandhi TK, Kandasamy K, Suresh S, Mohmood $R$, et al. An evaluation of human protein-protein interaction data in the public domain. BMC Bioinformatics. 2006;7 Suppl 5:S19.

36. Li Z, Li F, Ni M, Li P, Bo X, Wang S. Characterization the regulation of herpesvirus miRNAs from the view of human protein interaction network. BMC Syst Biol. 2011;5:93.

\section{Submit your next manuscript to BioMed Central and take full advantage of:}

- Convenient online submission

- Thorough peer review

- No space constraints or color figure charges

- Immediate publication on acceptance

- Inclusion in PubMed, CAS, Scopus and Google Scholar

- Research which is freely available for redistribution 